ARTICLE

https://doi.org/10.1038/s41467-019-09781-y

\title{
Electroluminescence from multi-particle exciton complexes in transition metal dichalcogenide semiconductors
}

\author{
Matthias Paur ${ }^{1,4}$, Aday J. Molina-Mendoza (1) ${ }^{1,4}$, Rudolf Bratschitsch ${ }^{2}$, Kenji Watanabe (1) ${ }^{3}$, Takashi Taniguchi ${ }^{3}$ \& \\ Thomas Mueller ${ }^{1}$
}

Light emission from higher-order correlated excitonic states has been recently reported in hBN-encapsulated monolayer $\mathrm{WS}_{2}$ and $\mathrm{WS}_{2}$ upon optical excitation. These exciton complexes are found to be bound states of excitons residing in opposite valleys in momentum space, a promising feature that could be employed in valleytronics or other novel optoelectronic devices. However, electrically-driven light emission from such exciton species is still lacking. Here we report electroluminescence from bright and dark excitons, negatively charged trions and neutral and negatively charged biexcitons, generated by a pulsed gate voltage, in hexagonal boron nitride encapsulated monolayer $\mathrm{WSe}_{2}$ and $\mathrm{WS}_{2}$ with graphene as electrode. By tailoring the pulse parameters we are able to tune the emission intensity of the different exciton species in both materials. We find the electroluminescence from charged biexcitons and dark excitons to be as narrow as $2.8 \mathrm{meV}$.

\footnotetext{
${ }^{1}$ Vienna University of Technology, Institute of Photonics, Gußhausstraße 27-29, 1040 Vienna, Austria. ${ }^{2}$ Institute of Physics and Center for Nanotechnology, University of Münster, Wilhelm-Klemm-Strasse 10, 48149 Münster, Germany. ${ }^{3}$ National Institute for Materials Science, 1-1 Namiki, Tsukuba 305-0044, Japan. ${ }^{4}$ These authors contributed equally: Matthias Paur, Aday J. Molina-Mendoza. Correspondence and requests for materials should be addressed to A.J.M-M. (email: aday.molina-mendoza@tuwien.ac.at) or to T.M. (email: thomas.mueller@tuwien.ac.at)
} 
M onolayer transition metal dichalcogenide (TMD) semiconductors provide a unique platform to study light-matter interaction and many-body effects at the atomic scale. The strong Coulomb interaction in these materials leads to the formation of tightly bound electron-hole pairs (excitons) with binding energies of hundreds of millielectronvolts ${ }^{1-3}$. Moreover, excitons residing in the two different valleys at the $\mathrm{K}$ points of the Brillouin zone can also interact ${ }^{4-}$ ${ }^{10}$, giving rise to four- and five-particle states, which have been recently identified in tungsten diselenide ( $\left.\mathrm{WSe}_{2}\right)$ as neutral and charged biexcitons ${ }^{11-14}$, as well as biexcitons in $\mathrm{WS}_{2}{ }^{15,16}$. The radiative emission from such exciton complexes can further be tuned by an external electric field, modifying the doping of the material and thus favoring the formation of either charged or neutral complexes. This rich and complex excitonic scenario could be exploited to develop applications such as valleytronics and (quantum) optoelectronic devices, especially in the case of electrically-driven light emitters. Electroluminescence (EL) from monolayer TMDs has been widely studied in the past, especially at room temperature, where the emission originates from neutral excitons ${ }^{17-26}$. At cryogenic temperatures, the emission can also be due to localized exciton states or negatively charged trions ${ }^{27-33}$. However, electrically driven emission from more complex exciton species has not yet been observed.

Here, we report on EL from monolayer $\mathrm{WSe}_{2}$ and $\mathrm{WS}_{2}$ by pulsed transient $\mathrm{EL}^{34}$, which triggers the formation of exciton complexes and thus their light emission. The high sample quality, enabled by encapsulating the monolayer semiconductor in hexagonal boron nitride $(\mathrm{hBN})$, allows for the observation of EL from multi-particle exciton complexes, including neutral and negatively charged biexcitons, with narrow emission linewidths down to $\sim 2.8 \mathrm{meV}$ (full-width-at-half-maximum (FWHM)). Furthermore, the formation of different exciton species is tunable by tailoring of the electrical pulse parameters. Our results provide crucial insights into electrically-driven light emission in monolayer TMDs and open a new route to applications in novel optoelectronic devices.

\section{Results}

Sample structure and light emission at room temperature. The sample structure used to study EL in monolayer TMDs is schematically depicted in Fig. 1a. A microscope image of the $\mathrm{WS}_{2}$ sample presented in this article is shown in Fig. $1 \mathrm{~b}$ (a microscope image of the $\mathrm{WSe}_{2}$ sample is shown in Supplementary Fig. 1): the monolayer TMD (namely $\mathrm{WSe}_{2}$ or $\mathrm{WS}_{2}$ ) and graphene are encapsulated in between two multilayer hBN flakes. The graphene layer is used as electrical contact to the monolayer semiconductor. Part of the graphene flake is left un-encapsulated in order to make an electrical contact with a $\mathrm{Pd} / \mathrm{Au}$ electrode. The top $\mathrm{hBN}$ serves as encapsulation ${ }^{35}$ to avoid environment related effects (residues left after sample processing, adsorbents) on the light emission from TMDs, and the bottom hBN also provides an insulating layer to apply a gate voltage to the sample by means of a prepatterned $\mathrm{Ti} / \mathrm{Au}$ back-gate electrode, on top of which the entire heterostructure is placed. Graphene was chosen as electrode for two reasons: (i) it is a layered material that can be easily integrated in a van der Waals heterostructure, and (ii) it has been shown that charge carrier injection from graphene for transient EL is more efficient than from standard metals ${ }^{34}$, resulting in enhanced emission. Details of the sample fabrication can be found in the Methods section.

This sample structure (metal-insulator-semiconductor-graphene) allows for electrically driven light emission by the recently reported method of transient EL in transition metal dichalcogenides $^{34}$, in which light emission is achieved by alternated injection of electrons and holes into the semiconductor by means of a pulsed gate voltage. In Fig. 1c we show band diagrams that schematically depict the emission process. The shape of the ( $\sim \mathrm{ns}$ long) electrical pulses used for EL is schematically shown in the inset of Fig. 1c. The gate voltage sweeps from a negative value $\left(V_{0}\right)$, where the Fermi level in graphene is below the Dirac point and holes are accumulated in the valence band of the semiconductor (Fig. 1c left), to a positive value $\left(V_{1}\right)$, where the Fermi level in graphene shifts upwards in energy and electrons are injected into the conduction band (Fig. 1c right). The steep (lateral) band bending in the TMD due to the large voltage drop at the Schottky contact leads to a transient tunneling current. As a result, injected electrons diffuse in the semiconductor while holes (radiatively) recombine with incoming electrons or partially drift out through the contact ${ }^{34}$. This process is known as transient EL, as the injected carriers recombine only during the rise and fall periods of the pulse. The light generation can be depicted from Fig. 1d, where we show an optical microscopy picture of the sample presented in Fig. 1b, with a clear red-orangish light emission originating from the $\mathrm{WS}_{2}$ flake.

The samples are characterized at room temperature by their PL spectrum (Fig. 1e) upon optical excitation with a continuouswave laser $\left(\lambda=532 \mathrm{~nm}, P_{\mathrm{d}}=2 \times 10^{3} \mathrm{Wcm}^{-2}\right)$, where we observe an emission maximum centered at $\sim 1.653 \mathrm{eV}$ for $\mathrm{WSe}_{2}$ and at $\sim 2.003 \mathrm{eV}$ in the case of $\mathrm{WS}_{2}$, corresponding to their respective free exciton emission (comparison between spatial resolution of PL and EL is presented in Supplementary Figs. 1a and 2). Similar spectra are observed when an AC voltage is applied between the back-gate and the graphene layer, inducing transient EL near the interface between the TMD monolayer and graphene.

Electro- and photoluminescence at $\boldsymbol{T}=\mathbf{5} \mathrm{K}$. At room temperature, only one emission maximum can be identified in the PL and EL spectra of both $\mathrm{WSe}_{2}$ and $\mathrm{WS}_{2}$. However, the scenario becomes more complex when the sample is studied at cryogenic temperatures. At $T=5 \mathrm{~K}$ different emission peaks can now be distinguished in the EL spectra of both materials. In $\mathrm{WSe}_{2}$ (Fig. 2a), apart from the well-known bright exciton $\left(\mathrm{X}^{0}\right)$ centered at $1.7315 \mathrm{eV}$, the EL spectrum reveals the emission lines associated with other exciton complexes, previously observed upon optical excitation ${ }^{11-14}$. On one hand, we are able to distinguish the two negatively charged trions at 1.7007 and $1.6921 \mathrm{eV}$, attributable to the intravalley $\left(\mathrm{X}_{1}^{-}\right)$and intervalley trions $\left(\mathrm{X}_{2}^{-}\right)$, respectively ${ }^{36}$, and the spin-forbidden dark exciton $\left(\mathrm{X}^{\mathrm{D}}\right)$ at $1.6864 \mathrm{eV}$, which is the two-particle ground state exciton $6,37,38$. Furthermore, the neutral biexciton (XX) centered at $1.7121 \mathrm{eV}$, a four-particle complex, is formed from a long-lived dark (spinforbidden) and a bright (spin-allowed) exciton, while the charged biexciton centered at $1.6808 \mathrm{eV}\left(\mathrm{XX}^{-}\right)$, a five-particle complex, is formed from a negatively charged trion and a neutral exciton in different $\mathrm{K}$ and $\mathrm{K}^{\prime}$ valleys ${ }^{8,11-14}$. It is worth mentioning that the pulsed EL from $\mathrm{WSe}_{2}$ shows a narrow emission linewidth of $\sim 2.8$ meV FWHM only for both the charged biexciton and the dark exciton. Finally, we also observe a broad peak at $\sim 1.675 \mathrm{eV}$ which may be assigned to a positively or negatively charged dark trion $\left(\mathrm{X}^{\mathrm{D} \pm}\right)^{39}$. At energies lower than $\sim 1.67 \mathrm{eV}$, additional emission peaks (labelled $\mathrm{L}_{1}, \mathrm{~L}_{2}$, and $\mathrm{L}_{3}$ ) emerge that most probably stem from localized states ${ }^{37,40-44}$. In Table 1 , we summarize the energy of the different exciton complexes with respect to the bright exciton $\mathrm{X}^{0}$.

A similar scenario is uncovered in the EL spectrum of $\mathrm{WS}_{2}$, shown in Fig. $2 \mathrm{~b}$ (Table 1 provides again a summary of the exciton energies). The bright exciton $\mathrm{X}^{0}$ appears at an energy of $2.075 \mathrm{eV}$, as well as the charged trions $\mathrm{X}_{1}^{-}$and $\mathrm{X}_{2}^{-}$at 2.045 and $2.038 \mathrm{eV}$, respectively. The peak appearing at $2.023 \mathrm{eV}$ has previously been 
a

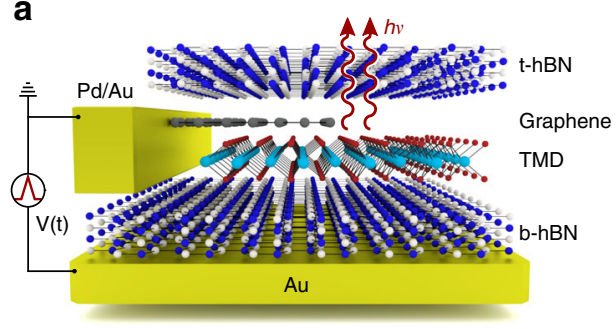

C

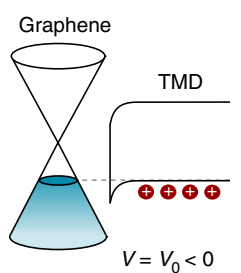

Graphene
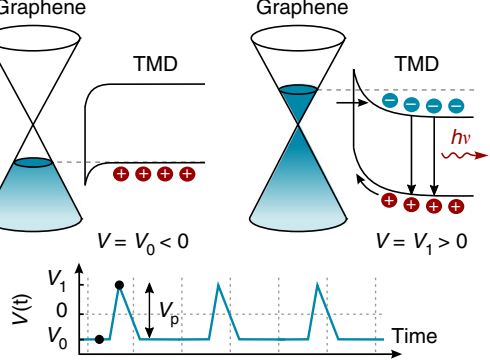

b

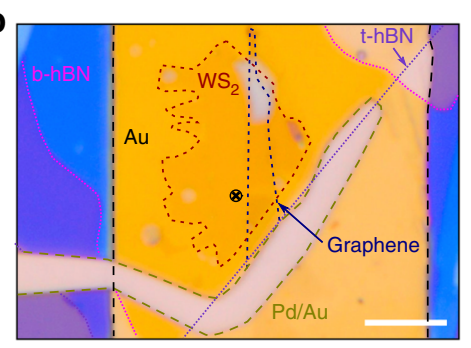

d

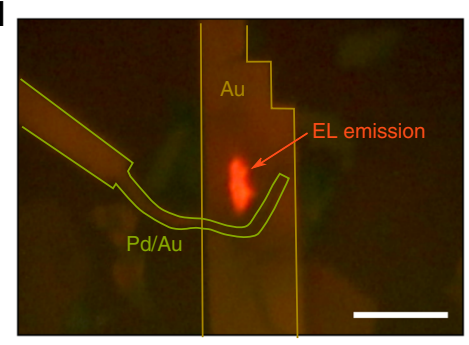

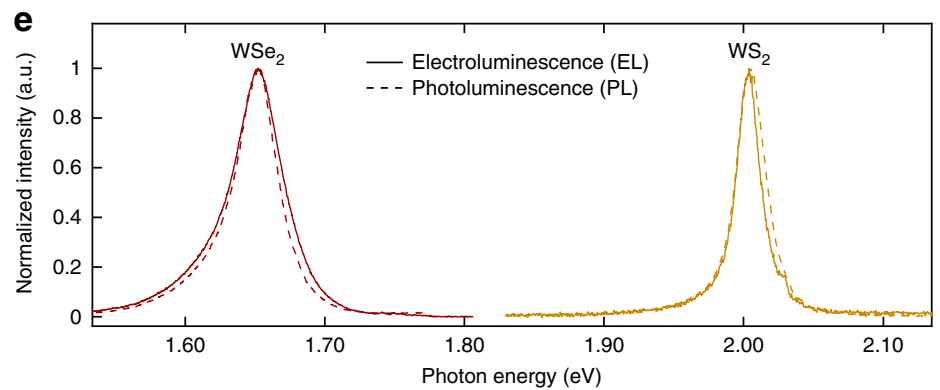

Fig. 1 Sample structure and light emission at $T=300 \mathrm{~K}$. a Schematic drawing of the sample. The monolayer TMD and graphene are sandwiched between two multilayer hBN flakes. b Optical microscope image of a typical sample, in this case based on $\mathrm{WS}_{2}$. The cross marks the position at which the PL spectra were taken. Scale bar, $5 \mu \mathrm{m}$. c Band diagram of transient EL. On the left, the pulse voltage is at a negative value, while on the right it is at a positive value. The bottom picture schematically depicts the shape of the pulse. d Optical microscopy image of the sample while emitting light. The EL emission is generated near the interface between graphene and monolayer $\mathrm{WS}_{2}$ due to carrier diffusion from graphene. Scale bar, $20 \mu \mathrm{m}$. e Photoluminescence (PL) and electroluminescence (EL) emission spectrum (excitation $\lambda=532 \mathrm{~nm}, P_{\mathrm{d}}=2 \times 10^{3} \mathrm{Wcm}^{-2}$ ). The EL spectrum closely matches the PL spectrum

associated to a biexciton 15,16 , although its charged or neutral nature remains still unclear. Here we anticipate that it is due to a negatively charged biexciton $\left(\mathrm{XX}^{-}\right)$, since its peak height (emission intensity hereafter) increases when a positive gate voltage is applied to the sample, as will be discussed later. Furthermore, we are able to observe additional emission lines corresponding to a dark exciton $\left(\mathrm{X}^{\mathrm{D}}\right)$, centered at $2.028 \mathrm{eV}$ (only observed so far in the PL of non-encapsulated samples ${ }^{45}$ ) and to a neutral biexciton (XX), centered at $2.056 \mathrm{eV}$, which, to our knowledge, has not been previously reported. Importantly, the latter two exciton complexes are hardly visible in the PL spectrum, but can be clearly resolved in the respective EL. To our knowledge, this is the first evidence of EL of such multi-particle complexes in both $\mathrm{WSe}_{2}$ and $\mathrm{WS}_{2}$.

The rich EL scenario in our samples, based on charged and neutral excitonic complexes, motivates the operation of such heterostructures as tunable light emitters by just tailoring the pulse parameters. By shifting the offset of the pulsed voltage, it is possible to create either an electron-rich or a hole-rich environment in the $2 \mathrm{D}$ semiconductor, consequently enhancing or diminishing EL from the different exciton complexes in a similar way as it is commonly observed in gated PL measurements. To this end, according to the schematic pulse diagram shown in Fig. 1c, the EL experiment is performed as follows (see also Supplementary Fig. 3): the pulse amplitude $V_{\mathrm{p}}=\left|V_{1}-V_{0}\right|$ is kept constant in all measurements, while the offset voltage $V_{0}$ is varied over a certain range. In this situation, an offset of $V_{0}=0$ indicates that the voltage applied to the back-gate sweeps in a pulse cycle from 0 to $V_{\mathrm{p}}$, while with an offset of $V_{0}=-V_{\mathrm{p}}$, the voltage applied to the back-gate sweeps from $-V_{\mathrm{p}}$ to 0 . Therefore, in the former case an electron-rich $\left(\mathrm{p}^{-} / \mathrm{n}^{+}\right)$environment is induced in the semiconductor, while in the latter the injected charge carrier density will be hole-rich $\left(\mathrm{p}^{+} / \mathrm{n}^{-}\right)$. Finally, in the symmetric case, $V_{0}=-V_{\mathrm{p}} / 2$, the amount of injected electrons and holes is balanced $(\mathrm{p} / \mathrm{n})$. Note that this picture applies to the case of undoped devices with contacts that are equally transparent for both electrons and holes. In realistic samples the different regimes may occur at other offset voltages, but the qualitative picture remains the same.

In Fig. 2c, d we show colormaps representing the EL emission from $\mathrm{WSe}_{2}$ and $\mathrm{WS}_{2}$, respectively, for different $V_{0}$ values and with fixed pulse amplitude of $V_{\mathrm{p}}=7.0 \mathrm{~V}$. We observe in both materials that for higher offset values $\left(\mathrm{p}^{-} / \mathrm{n}^{+}\right.$region), i.e. higher electron injection, the $\mathrm{EL}$ is dominated by negatively charged exciton species such as $\mathrm{XX}^{-}, \mathrm{X}_{1}^{-}$, and $\mathrm{X}_{2}^{-}$, where the charged biexciton is the predominant emission and the free exciton is much weaker. For lower offset values ( $\mathrm{p} / \mathrm{n}$ region), the emission from negatively charge exciton complexes vanish and the spectrum is then dominated by neutral species such as the dark and bright excitons and neutral biexciton, as well as localized excitons. This indicates that the charge carrier injection is balanced between electrons and holes, favoring the formation of neutral excitons. Finally, when the offset voltage has a more negative value $\left(\mathrm{p}^{+} / \mathrm{n}^{-}\right.$region), the 
a

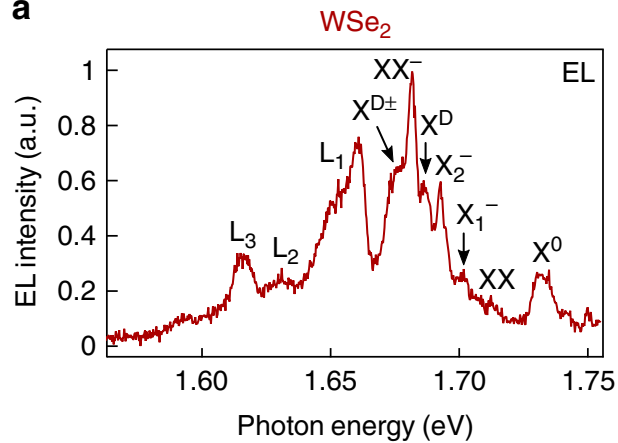

C
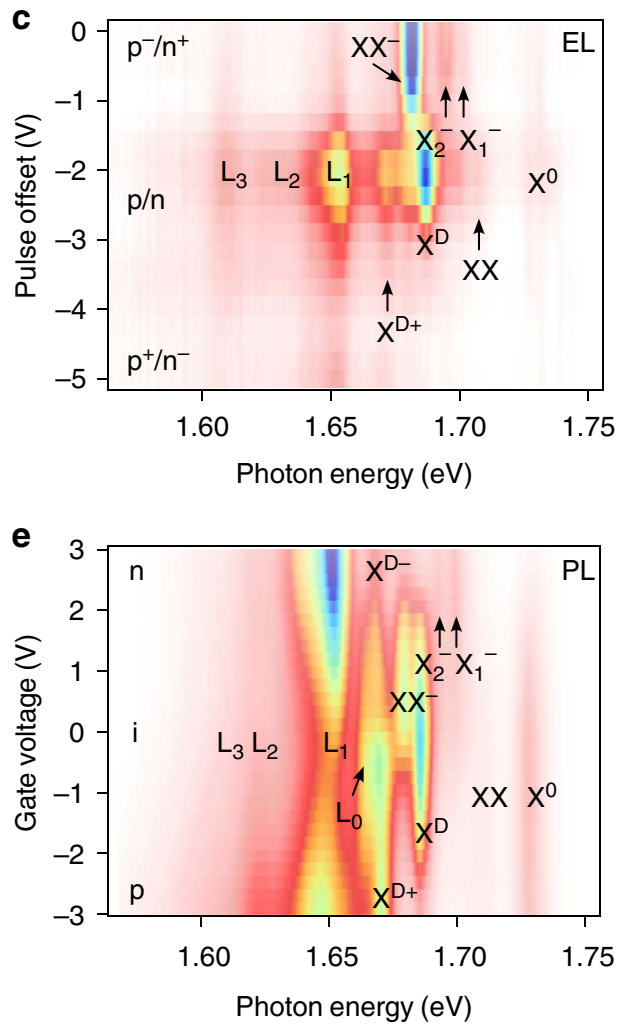

b

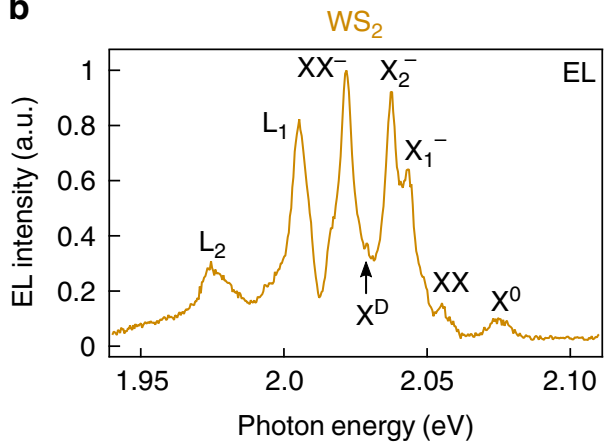

d
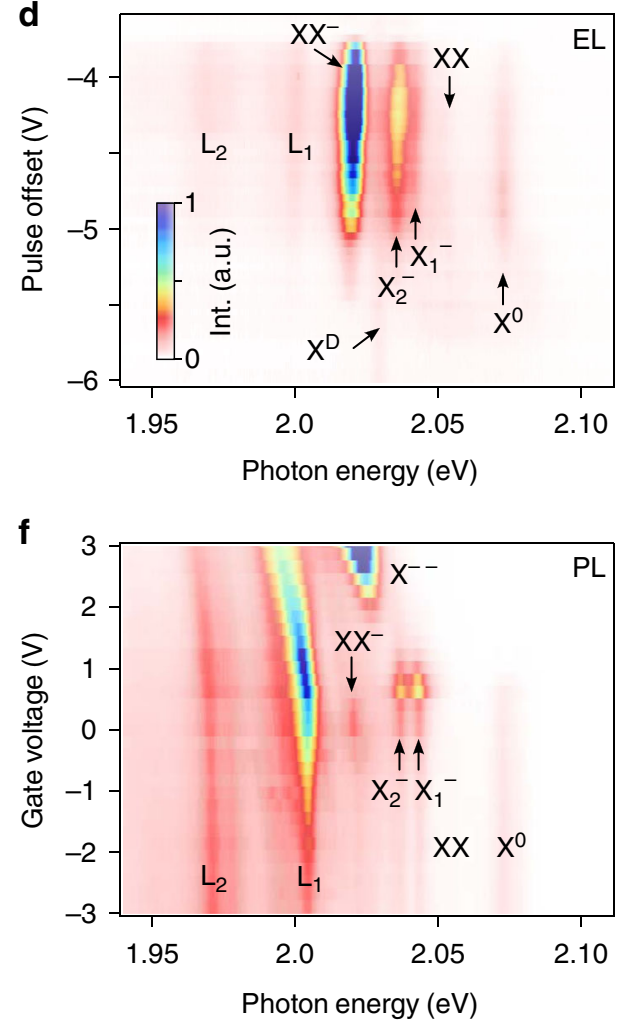

Fig. 2 Emission spectra and tunability at $T=5 \mathrm{~K}$. Pulsed EL from a WSe $e_{2}$ and $\mathbf{b} \mathrm{WS}_{2}$. In $\mathrm{WSe}_{2}$, a narrow emission (linewidth $\sim 2.8 \mathrm{meV} \mathrm{FWHM}$ ) is found for both the charged biexciton and the dark exciton. Colormap of EL spectra at $5 \mathrm{~K}$ plotted as function of pulse offset $V_{0}$ in $\mathbf{c} W \mathrm{We}_{2}$ and $\mathbf{d} W S_{2}\left(V_{p}=7.5 \mathrm{~V}\right)$. Colormap of charged doping control of PL emission in $\mathbf{e} \mathrm{WSe}_{2}$ and $\mathbf{f} \mathrm{WS}_{2}$, for different exciton species acquired at an excitation of $P_{\mathrm{d}}=5 \times 10^{3} \mathrm{Wcm}-2$

\section{Table 1 Energy of the different exciton complexes with respect to the bright exciton $\mathrm{X}^{0}$ appearing in the EL spectra}

\begin{tabular}{|lllllll} 
& $\mathbf{X}^{\mathbf{0}}(\mathbf{e V})$ & $\begin{array}{l}\mathbf{X}^{\mathbf{0}} \\
\mathbf{X X} \\
(\mathbf{m e V})\end{array}$ & $\begin{array}{l}\mathbf{X}^{\mathbf{0}}-\mathbf{X}_{\mathbf{1}}^{-} \\
(\mathbf{m e V})\end{array}$ & $\begin{array}{l}\mathbf{X}^{\mathbf{0}}-\mathbf{X}_{\mathbf{2}}^{-} \\
(\mathbf{m e V})\end{array}$ & $\begin{array}{l}\mathbf{X}^{\mathbf{0}}-\mathbf{X}^{\mathbf{D}} \\
(\mathbf{m e V})\end{array}$ & $\begin{array}{l}\mathbf{X}^{\mathbf{0}}-\mathbf{X X} \\
-(\mathbf{m e V})\end{array}$ \\
\hline $\mathrm{WSe}_{2}$ & 1.7315 & 19.4 & 30.8 & 39.4 & 45.1 & 50.7 \\
$\mathrm{WS}_{2}$ & 2.0752 & 19.2 & 30.2 & 36.6 & 46.36 & 52.4 \\
\hline
\end{tabular}

induced charge density in the semiconductor is then hole-rich and the emission from positively charged species such as the positive dark trion in $\mathrm{WSe}_{2}$ becomes stronger. We are not able to resolve positively charged trions in EL neither in $\mathrm{WSe}_{2}$ or $\mathrm{WS}_{2}$, possibly because the induced hole concentration is too low to favor trion formation.

The tunable EL can be compared to the respective PL colormaps (Fig. 2e, f), where the PL emission is measured for different DC gate voltages $\left(V_{g}\right)$. In a similar manner as for EL, negative exciton species become the dominant emission for positive applied gate voltages $\left(V_{g}>0\right)$, i.e. for $\mathrm{n}$-doping, although in the PL spectra we are able to additionally resolve the negative dark trion in $\mathrm{WSe}_{2}$ and the double-negatively charged trion $\left(\mathrm{X}^{--}\right)$, the next charging state of the trion ${ }^{13}$, in $\mathrm{WS}_{2}$. In the $\mathrm{WS}_{2}$ spectra we also observe $\mathrm{XX}^{-}$ emission only in the $\mathrm{n}$-doped region, supporting the negatively charged nature of this biexciton as concluded above from the EL measurements. Around $V_{g}=0$, the negatively charged species vanish and only the neutral excitons and biexciton, as well as the localized states, show emission, again in accordance with the EL spectra. Here it is worth noting that we are not aware of any previous reports on the PL emission of neutral biexcitons in $\mathrm{WS}_{2}$, which we are able to resolve both in EL and PL. Finally, for $V_{g}<0$, the positive dark trion appears in the $\mathrm{WSe}_{2} \mathrm{PL}$ spectrum, but not in the one of $\mathrm{WS}_{2}$, suggesting that $\mathrm{WS}_{2}$ is unintentionally $\mathrm{n}$-doped and we therefore cannot achieve sufficient p-doping in this material. We refer the reader to the Supplementary Figs. 4 and 5 

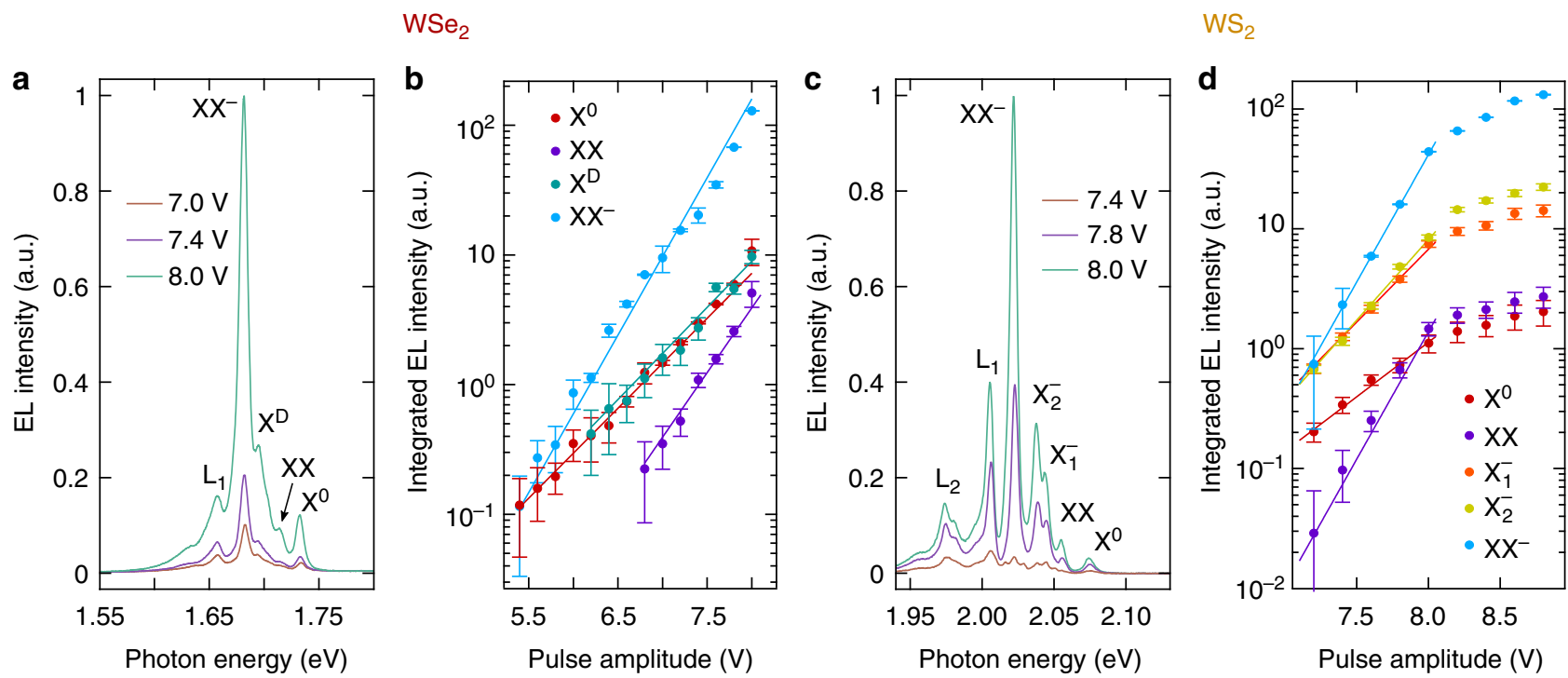

Fig. 3 EL intensity dependence with the excitation power. a Pulsed EL emission spectra of WSe 2 for different pulse amplitudes and fixed offset voltage. b Semi-logarithmic plot of the integrated EL intensity as a function of the pulse amplitude. The circles represent the experimental data, while the lines depict the fit to an exponential function as discussed in the main text. c, d Same as in $\mathbf{a}, \mathbf{b}$ but for $\mathrm{WS}_{2}$

for a further analysis of the PL spectra as a function of the backgate voltage.

Discrimination between different multi-particle excitons. The electrical tunability of the EL and PL spectra permits the discrimination between charged and neutral exciton species. Whether they are formed by two, three, four or five particles cannot be distinguished by this method. We therefore studied the dependence of the emission intensity with the excitation intensity. While the emission intensities of dark and bright excitons, composed by one electron and one hole, follow a linear dependence with the excitation power, biexcitons are expected to show a quadratic behavior ${ }^{11-16}$. In Supplementary Figs. 6, 7, and 8 we show the PL spectra of $\mathrm{WSe}_{2}$ and $\mathrm{WS}_{2}$, at different excitation powers and fits of the respective intensities to the power-law $I_{P L} \propto P^{\alpha}$, where $I_{P L}$ is the integrated PL emission and $P$ the incident laser power. These fits yield the exponent values $\alpha=1.01$ for $\mathrm{X}^{0}$, $\alpha=1.82$ for $\mathrm{XX}$, and $\alpha=1.44$ for $\mathrm{XX}^{-}$in $\mathrm{WSe}_{2}$, and similar values in $\mathrm{WS}_{2}$ (see Supplementary Table 1). The deviation from the quadratic growth for the charged biexciton can be attributed to the lack of equilibrium between exitonic states 46,47 .

We have also studied the dependence of the emission intensity on the excitation power in EL by keeping a fixed offset voltage and changing the electrical pulse amplitude. For increasing pulse amplitude, the Fermi level shifts deeper in the conduction band and, therefore, more charge carriers are injected, resulting in increased EL emission. The measured spectra are shown in Fig. 3a, c for $\mathrm{WSe}_{2}$ and $\mathrm{WS}_{2}$, respectively, and the corresponding plots of EL intensities against pulse amplitude are presented in Fig. 3b, d. On a semi-logarithmic scale, the dependence of EL intensity versus voltage shows a linear behavior for all exciton species. This observation motivates us to empirically model the EL intensity by the expression $I_{E L} \propto \exp \left(\alpha \kappa V_{\mathrm{p}}\right)$, where $V_{\mathrm{p}}$ is the applied voltage, $\kappa$ is a constant related to the injected carrier concentration in the $2 \mathrm{D}$ semiconductor, and $\alpha$ is a coefficient that indicates the nature of the exciton, as in the PL measurements. We fix $\kappa$ to fit the data for the neutral exciton $\mathrm{X}^{0}$ with $\alpha \equiv 1$, which then serves as reference for determining the $\alpha$ values of all other exciton species.
The fit of the measurement data to this empirical expression yields for $\mathrm{WSe}_{2}$ a coefficient of $\alpha=1.75$ for $\mathrm{XX}^{-}$, which is significantly larger than that of the $\mathrm{X}^{0}$ and $\mathrm{X}^{D}$ excitons $(\alpha \equiv 1$ and $\alpha=1.03$, respectively) and it is close to a quadratic dependence. For the neutral biexciton XX we obtain a coefficient of $\alpha=1.44$. For $\mathrm{WS}_{2}$, the coefficients for $\mathrm{X}^{0}, \mathrm{X}_{1}^{-}$, and $\mathrm{X}_{2}^{-}$are $\alpha \equiv 1, \alpha=1.32$, and $\alpha=1.33$, respectively, and $\alpha=2.33$ and $\alpha=2.31$ for the neutral biexciton XX and charged biexciton $\mathrm{XX}^{-}$. In Supplementary Table 1 we summarize the obtained coefficients for the different exciton complexes. The emission of all exciton species in $\mathrm{WS}_{2}$ saturate at voltage amplitudes larger than $\sim 8 \mathrm{~V}$, in accordance with measurements performed at room temperature by Lien et al. ${ }^{34}$. We do not observe such saturation in $\mathrm{WSe}_{2}$, most probably because of the lower peak voltage applied in these measurements. Finally, we present EL spectra for both $\mathrm{WSe}_{2}$ and $\mathrm{WS}_{2}$ at different pulse repetition rates in Supplementary Fig. 9.

\section{Discussion}

In summary, we have studied transient EL of monolayer TMD semiconductors (in particular $\mathrm{WSe}_{2}$ and $\mathrm{WS}_{2}$ ) sandwiched between two layers of $\mathrm{hBN}$ and one graphene sheet at low temperatures. The high material quality of our samples, together with the strong Coulomb interaction between electrons and holes in 2D semiconductors, permits the observation of electrically driven light emission of higher-order correlated excitonic states, including bright and dark excitons, negatively charged trions and neutral and negatively charged biexcitons. By tailoring the pulse parameters, it is possible to create either an electron- or a holerich environment in the $2 \mathrm{D}$ semiconductor, consequently favoring the enhanced or diminished EL from the different exciton species. Our technique extends and complements gate-dependent PL spectroscopy and will enable further investigations of manybody phenomena in 2D materials. From an applied point of view, our devices may find application as wavelength tunable light emitters or furnish new opportunities for quantum light sources, e.g. by quantum-confinement of electrically induced biexcitons.

\section{Methods}

Sample fabrication. Bulk WSe $e_{2}$ (grown by vapor phase transport; see ref. ${ }^{48}$ for details) and $\mathrm{WS}_{2}$ (purchased from HQ Graphene), graphite and hBN were exfoliated by an adhesive tape and transferred onto a $\mathrm{SiO}_{2}$ substrate thermally grown 
on Si. The substrates were cleaned by an acetone/isopropanol bath and oxygen plasma treatment. They were then annealed at $300^{\circ} \mathrm{C}$ for $2 \mathrm{~h}$ in a dry-air atmosphere prior to the transfer of the exfoliated materials to remove possible adsorbates. The tape was peeled-off afterward and the atomically-thin flakes were selected with optical microscopy. A pick-up and place technique ${ }^{49}$ was used to pick up the flakes by means of a polypropylene carbonate/polydimethylsiloxane (PPC/ PDMS) stamp, starting with the top hBN flake. The heterostructure (hBN/graphene $/ \mathrm{WSe}_{2} / \mathrm{hBN}$ ) was then transferred onto a Ti/Au $(5 / 100 \mathrm{~nm})$ electrode, prepatterned on $\mathrm{SiO}_{2} / \mathrm{Si}$ substrate. The entire exfoliation and transfer process was performed in a nitrogen-purged glovebox. The remaining PPC residues were removed by introducing the sample in an acetone/chloroform bath. Finally, an additional electrode was fabricated by means of electron-beam lithography and metal evaporation to make an electrical contact to the graphene flake ( $\mathrm{Pd} / \mathrm{Au}, 60 /$ $140 \mathrm{~nm})$.

PL and EL measurements. For EL measurements a pulse generator (Agilent $8114 \mathrm{~A}$ ) in combination with a source-meter unit (Keithley 2612A) was used. The pulses were applied to the bottom electrode, while the top contact was grounded. Optical and electrical investigations were carried out in a liquid-helium exchange gas cryostat (Oxford Instruments) under high vacuum $\left(10^{-7} \mathrm{mbar}\right)$, integrated in a scanning micro-PL setup. The setup includes a dichroic mirror at $\lambda=550 \mathrm{~nm}$ to separate the PL emission from the excitation. PL and EL were collected using a $\times 50$ long working distance objective lens $(\mathrm{NA}=0.5)$. In PL experiments, the sample was optically excited non-resonantly using a continuous-wave $532 \mathrm{~nm}$ laser; the diameter of the laser beam on the sample was $\sim 1 \mu \mathrm{m}$. The PL/EL emission was spectrally filtered with a monochromator (Horiba iHR320) and detected with a liquid nitrogen cooled charge-coupled device (CCD). EL imaging was performed by using a CCD camera (Thorlabs).

\section{Data availability}

The data that support the findings of this study are available from the authors upon reasonable request; see authors contributions for specific data.

Received: 6 December 2018 Accepted: 1 April 2019

Published online: 12 April 2019

\section{References}

1. Wang, G. et al. Colloquium: Excitons in atomically thin transition metal dichalcogenides. Rev. Mod. Phys. 90, 021001 (2018).

2. He, K. et al. Tightly bound excitons in monolayer WSe2. Phys. Rev. Lett. 113, 026803 (2014).

3. Chernikov, A. et al. Exciton binding energy and nonhydrogenic rydberg series in monolayer WS2. Phys. Rev. Lett. 113, 076802 (2014)

4. Mak, K. F., He, K., Shan, J. \& Heinz, T. F. Control of valley polarization in monolayer MoS2 by optical helicity. Nat. Nanotechnol. 7, 494-498 (2012).

5. Hao, K. et al. Neutral and charged inter-valley biexcitons in monolayer MoSe2. Nat. Commun. 8, 15552 (2017).

6. Zhang, X.-X. et al. Magnetic brightening and control of dark excitons in monolayer WSe2. Nat. Nanotechnol. 12, 883-888 (2017).

7. Robert, C. et al. Fine structure and lifetime of dark excitons in transition metal dichalcogenide monolayers. Phys. Rev. B 96, 155423 (2017).

8. Stevens, C. E. et al. Biexcitons in monolayer transition metal dichalcogenides tuned by magnetic fields. Nat. Commun. 9, 3720 (2018).

9. Berkelbach, T. C., Hybertsen, M. S. \& Reichman, D. R. Theory of neutral and charged excitons in monolayer transition metal dichalcogenides. Phys. Rev. B 88, 045318 (2013).

10. Zhang, X.-X., You, Y., Zhao, S. Y. F. \& Heinz, T. F. Experimental evidence for dark excitons in monolayer WSe2. Phys. Rev. Lett. 115, 257403 (2015).

11. Ye, Z. et al. Efficient generation of neutral and charged biexcitons in encapsulated WSe2 monolayers. Nat. Commun. 9, 3718 (2018)

12. $\mathrm{Li}, \mathrm{Z}$. et al. Revealing the biexciton and trion-exciton complexes in $\mathrm{BN}$ encapsulated WSe2. Nat. Commun. 9, 3719 (2018).

13. Barbone, M. et al. Charge-tuneable biexciton complexes in monolayer WSe2. Nat. Commun. 9, 3721 (2018).

14. Chen, S.-Y., Goldstein, T., Taniguchi, T., Watanabe, K. \& Yan, J. Coulombbound four- and five-particle intervalley states in an atomically-thin semiconductor. Nat. Commun. 9, 3717 (2018).

15. Shang, J. et al. Observation of excitonic fine structure in a $2 \mathrm{~d}$ transition-metal dichalcogenide semiconductor. ACS Nano 9, 647-655 (2015).

16. Nagler, P. et al. Zeeman splitting and inverted polarization of biexciton emission in monolayer WS2. Phys. Rev. Lett. 121, 57402 (2018).

17. Ross, J. S. et al. Electrically tunable excitonic light-emitting diodes based on monolayer WSe2 p-n junctions. Nat. Nanotechnol. 9, 268-272 (2014).
18. Pospischil, A., Furchi, M. M. \& Mueller, T. Solar-energy conversion and light emission in an atomic monolayer p-n diode. Nat. Nanotechnol. 9, 257-261 (2014).

19. Baugher, B. W. H., Churchill, H. O. H., Yang, Y. \& Jarillo-Herrero, P. Optoelectronic devices based on electrically tunable $\mathrm{p}-\mathrm{n}$ diodes in a monolayer dichalcogenide. Nat. Nanotechnol. 9, 262-267 (2014).

20. Frisenda, R., Molina-Mendoza, A. J., Mueller, T., Castellanos-Gomez, A. \& van der Zant, H. S. J. Atomically thin p-n junctions based on two-dimensional materials. Chem. Soc. Rev. 47, 3339-3358 (2018).

21. $\mathrm{Pu}, \mathrm{J}$. et al. A versatile and simple approach to generate light emission in semiconductors mediated by electric double layers. Adv. Mater. 29, 1606918 (2017).

22. Hong, X. et al. Ultrafast charge transfer in atomically thin MoS2/WS2 heterostructures. Nat. Nanotechnol. 9, 682-686 (2014).

23. Plechinger, G. et al. Excitonic valley effects in monolayer ws 2 under high magnetic fields. Nano Lett. 16, 7899-7904 (2016).

24. Plechinger, G. et al. Identification of excitons, trions and biexcitons in singlelayer WS2. Phys. status solidi - Rapid Res. Lett. 9, 457-461 (2015).

25. Cong, C., Shang, J., Wang, Y. \& Yu, T. Optical properties of 2D semiconductor WS2. Adv. Opt. Mater. 6, 1700767 (2018).

26. Withers, F. et al. WSe ${ }_{2}$ light-emitting tunneling transistors with enhanced brightness at room temperature. Nano Lett. 15, 8223-8228 (2015).

27. Clark, G. et al. Single defect light-emitting diode in a van der waals heterostructure. Nano Lett. 16, 3944-3948 (2016).

28. Palacios-Berraquero, C. et al. Atomically thin quantum light-emitting diodes. Nat. Commun. 7, 12978 (2016).

29. Schwarz, S. et al. Electrically pumped single-defect light emitters in WSe2. $2 D$ Mater. 3, 025038 (2016)

30. Zhang, Y. J., Oka, T., Suzuki, R., Ye, J. T. \& Iwasa, Y. Electrically switchable chiral light-emitting transistor. Science. 344, 725-728 (2014).

31. Wang, J., Verzhbitskiy, I. \& Eda, G. Electroluminescent devices based on 2D semiconducting transition metal dichalcogenides. Adv. Mater. 30, 1802687 (2018).

32. Palacios-Berraquero, C. et al. Large-scale quantum-emitter arrays in atomically thin semiconductors. Nat. Commun. 8, 15093 (2017).

33. Ye, Y. et al. Monolayer excitonic laser. Nat. Photonics 9, 733-737 (2015).

34. Lien, D.-H. et al. Large-area and bright pulsed electroluminescence in monolayer semiconductors. Nat. Commun. 9, 1229 (2018).

35. Cadiz, F. et al. Excitonic linewidth approaching the homogeneous limit in MoS2-based van der waals heterostructures. Phys. Rev. X 7, 021026 (2017).

36. Jones, A. M. et al. Excitonic luminescence upconversion in a two-dimensional semiconductor. Nat. Phys. 12, 323-327 (2016).

37. Wang, G. et al. In-plane propagation of light in transition metal dichalcogenide monolayers: optical selection rules. Phys. Rev. Lett. 119, 047401 (2017).

38. Zhou, Y. et al. Probing dark excitons in atomically thin semiconductors via near-field coupling to surface plasmon polaritons. Nat. Nanotechnol. 12, 856-860 (2017).

39. Liu, E. et al. Gate tunable dark trions in monolayer WSe2. Preprint at https:// arxiv.org/abs/1901.11043. (2019)

40. Tonndorf, P. et al. Single-photon emission from localized excitons in an atomically thin semiconductor. Optica 2, 347 (2015).

41. Srivastava, A. et al. Optically active quantum dots in monolayer WSe2. Nat. Nanotechnol. 10, 491-496 (2015).

42. Koperski, M. et al. Single photon emitters in exfoliated WSe2 structures. Nat. Nanotechnol. 10, 503-506 (2015).

43. Chakraborty, C., Kinnischtzke, L., Goodfellow, K. M., Beams, R. \& Vamivakas, A. N. Voltage-controlled quantum light from an atomically thin semiconductor. Nat. Nanotechnol. 10, 507-511 (2015).

44. He, Y.-M. et al. Single quantum emitters in monolayer semiconductors. Nat. Nanotechnol. 10, 497-502 (2015).

45. Molas, M. R. et al. Brightening of dark excitons in monolayers of semiconducting transition metal dichalcogenides. 2D Mater. 4, 021003 (2017).

46. Phillips, R. T., Lovering, D. J., Denton, G. J. \& Smith, G. W. Biexciton creation and recombination in a GaAs quantum well. Phys. Rev. B 45, 4308-4311 (1992).

47. Birkedal, D., Singh, J., Lyssenko, V. G., Erland, J. \& Hvam, J. M. Binding of quasi-two-dimensional biexcitons. Phys. Rev. Lett. 76, 672-675 (1996).

48. Tonndorf, P. et al. Photoluminescence emission and Raman response of monolayer MoS2, MoSe2, and WSe2. Opt. Express 21, 4908-4916 (2013).

49. Pizzocchero, F. et al. The hot pick-up technique for batch assembly of van der Waals heterostructures. Nat. Commun. 7, 11894 (2016).

\section{Acknowledgements}

We acknowledge financial support by the Austrian Science Fund FWF (START Y 539N16) and the European Union (grant agreement No. 785219 Graphene Flagship). A.J.M.-M. acknowledges financial support from the European Commission (Marie Sklodowska-Curie Individual Fellowships, OPTOvanderWAALS, grant ID 791536). M.P. 
acknowledges financial support from the doctoral college program "TU-D” funded by TU Vienna. K.W. and T.T. acknowledge support from the Elemental Strategy Initiative conducted by the MEXT, Japan and the CREST (JPMJCR15F3), JST.

\section{Author contributions}

A.J.M.-M. and T.M. conceived the project. M.P. and A.J.M.-M. fabricated the samples and performed the measurements. R.B. provided high-quality $\mathrm{WSe}_{2}$ crystals. T.T. and $\mathrm{K}$. W. synthetized the hBN crystal. M.P., A.J.M.-M. and T.M. wrote the manuscript with input from all the authors. All authors discussed the results and contributed to the manuscript.

\section{Additional information}

Supplementary Information accompanies this paper at https://doi.org/10.1038/s41467019-09781-y.

Competing interests: The authors declare no competing interests.

Reprints and permission information is available online at http://npg.nature.com/ reprintsandpermissions/
Journal peer review information: Nature Communications thanks Matteo Barbone and the other anonymous reviewers for their contribution to the peer review of this work.

Publisher's note: Springer Nature remains neutral with regard to jurisdictional claims in published maps and institutional affiliations.

\section{(c) (1)}

Open Access This article is licensed under a Creative Commons Attribution 4.0 International License, which permits use, sharing, adaptation, distribution and reproduction in any medium or format, as long as you give appropriate credit to the original author(s) and the source, provide a link to the Creative Commons license, and indicate if changes were made. The images or other third party material in this article are included in the article's Creative Commons license, unless indicated otherwise in a credit line to the material. If material is not included in the article's Creative Commons license and your intended use is not permitted by statutory regulation or exceeds the permitted use, you will need to obtain permission directly from the copyright holder. To view a copy of this license, visit http://creativecommons.org/licenses/by/4.0/.

(C) The Author(s) 2019 